\title{
EVALUACIÓN DE LA POLÍTICA INDUSTRIAL DURANTE EL PERIODO DE APERTURA ECONÓMICA EN MÉXICO
}

\author{
Industrial policy evaluation during \\ economic liberalization in Mexico \\ Cuauhtémoc Calderón Villarreal ${ }^{1}$, Belem Iliana Vázquez² ${ }^{2}$ Laura Ivonne López Valdez ${ }^{3}$
}

Fecha de recepción: 25 junio de 2017

Fecha de aceptación: 20 de agosto de 2017

1- Nombre: Cuauhtémoc Calderón Villarreal. Nacionalidad: Mexicana. Grado: Doctor . Especialización:Ciencias Económicas. Adscripción: El Colegio de la Frontera Norte, A.C. Correo Electrónico: ccalderon17@outlook.com

2- Nombre: Belem Iliana Vázquez. Nacionalidad: Mexicana. Grado: Doctora. Especialización: Economía. Adscripción: El Colegio de la Frontera Norte, A.C. Correo Electrónico:belem@colef.mx

3- Nombre: Laura Ivonne López Valdez. Nacionalidad: Mexicana. Grado: Maestría. Especialización Economía Aplicada. Adscripción: Colegio de la Frontera Norte. Correo Electrónico: llopezv07@gmail.com 


\section{Resumen}

La Política Macroeconómica en México, desde mediados de los noventa, favoreció la estabilidad de precios y del tipo de cambio. Un rasgo característico fue la ausencia de una política industrial activa promotora del desarrollo económico nacional, en su lugar se aplicó una política comercial. El objetivo de esta investigación es evaluar si la política comercial aplicada, de apertura indiscriminada, logró impulsar el crecimiento de la producción manufacturera, las exportaciones y la formación bruta de capital. Se estimaron vectores de corrección de error con datos de 1995 a 2015. Los resultados indican que ningún instrumento de política comercial contribuyó al crecimiento de las manufacturas, pero hay evidencia de que el control de precios, las exportaciones y la IED facilitaron la formación bruta de capital y el dualismo económico. Esto implica que el sector orientado hacia afuera fue el más beneficiado, pero no generaron crecimiento en el resto de la economía. Es necesario considerar la aplicación de una política industrial activa que financie y promueva la producción dirigida al mercado interno.

Palabras clave: Política industrial, apertura, Producto Interno Bruto, capital.

\section{Abstract}

The Mexican macroeconomic policy has favored the stability of prices and the exchange rate since the early nineties. This has resulted in the absence of an active industrial policy that could promote economic development; instead a commercial policy was applied. The goal of this research is to evaluate whether or not this commercial policy contributed to raise growth rates of manufactures production, exports and gross fixed investment. We estimated a vector error correction mechanism with data from 1995 to 2015. None of the variables used by the commercial policy was significant as explicative of manufactures production growth. However, prices, exports and FDI contributed to increase gross fixed investment growth. The implication of the results is that outward oriented activities achieved better growth rates, however they had little effect on the rest of the economy. It is necessary to consider the application of a more active industrial policy in order to improve domestic production to satisfy domestic demand.

Key words: Policy, industry, openness, Gross Domestic Product, capital. 


\section{Introducción}

$\mathrm{L}$ a emergencia del proteccionismo en los Estados Unidos, nos obliga a reconsiderar la importancia del Estado como agente promotor del desarrollo económico en México. Es necesario reconsidera su papel dados los desafíos que enfrenta el país frente a los cambios en el mercado mundial. En estos momentos es pertinente evaluar si la política comercial de apertura total, que tiene ya más de treinta años en México, está agotada y requiere ser mejorada o sustituida por una política comercial e industrial cuya orientación sea hacia el mercado interno.

En sentido estricto la industrialización reside en la aplicación de una serie de instrumentos de política económica activa que estimulen, de manera directa, la formación de capital o acumulación de capital. Para ello, se requiere la acción directa del Estado en sectores económicos estratégicos, en donde el capital privado nacional sea incapaz de promover el desarrollo económico y de competir con el capital transnacional.

La utilización de la política industrial para promover el crecimiento económico fue duramente criticada en las décadas de los setenta y ochenta, ya que se asociaba el bajo desempeño económico con la ineficiencia de las políticas industriales promovidas por el Estado (Little, Scitovsky y Scott, 1970; Krueger, 1974; Balassa, 1982). Sin embargo, algunos estudios demostraron que el logro de las altas tasas de crecimiento alcanzadas por los países del Este Asiático se debía en parte al fuerte intervencionismo del Estado el cual, a través de una política industrial selectiva (y activa), otorgaba incentivos económicos a cambio de que las empresas beneficiadas alcanzaran objetivos de desempeño (Amsden, 1994; Fishlow, 1994). En un análisis comparativo de un grupo de países del Este Asiático con otro de América Latina, Shapiro (2007) encontró que entre 1980 y 2000, el valor agregado de las manufacturas en el Este de Asia había aumentado 9.1\% mientras que en América Latina fue de 1.4\%. Una de las razones que Shapiro ofrece es que los países asiáticos contaban con una fuerte tradición de aplicar la investigación y desarrollo y tecnología en sus procesos productivos, de tal manera que permitieron que empresas locales y extranjeras generaran derramas en el resto de la economía. Por el otro lado, en América Latina, el crecimiento de las manufactureras se basó, en gran parte, en la ventaja que otorgaba la disponibilidad de recursos, mientras la tecnología mantuvo una baja participación en la estructura productiva (Lall, Albaladejo y Moreira, 2004). Desde nuestro punto de vista, el bajo crecimiento se explica por la ausencia de la intervención del Estado, por el predominio del mercado externo, derivado del concepto de ventajas comparativas, que ha destruido las capacidades endógenas de crecimiento de los países de América Latina.

Teóricamente, el concepto de política industrial se fundamenta en la teoría estructuralista o neoestructuralista del desarrollo económico (desarrollada a partir de la teoría proteccionista de List y las teorías de Kalecki de corte postkeynesiano), en la cual la participación del Estado es crucial, ya que actúa como creador de las capacidades productivas de la economía, como promotor del cambio estructural y regulador de la economía ante las fallas del mercado. El diseño de la política industrial tiene diferentes enfoques según los instrumentos utilizados para alcanzar los objetivos de la industrialización. En la corriente poskeynesiana, se asume que las economías modernas están sujetas a importantes fluctuaciones en el producto, por lo que los gobiernos deben centrarse en políticas que estimulen la demanda y oferta agregada y el mercado interno, (Howells, 2012). En este sentido, lo ideal para una estrategia industrial 
es acelerar la inversión y dar prioridad a inversiones nuevas ya que esto permite renovar y modernizar el capital (López-Gallardo, 1999).

De acuerdo con la política industrial promotora del desarrollo económico de la década de los cuarenta, en el marco de una economía cerrada, era necesario utilizar el excedente generado por la agricultura para impulsar el crecimiento hacia adentro de la economía; primero, industrializando al sector de bienes ligeros y segundo al del sector de bienes pesados. En especial, buscaba la sustitución de las importaciones de ambos sectores con la finalidad de generar capacidades productivas autónomas en la economía nacional. Se consideraba que la industrialización era la base del desarrollo económico. En esta tesitura Kaldor (1966) argumentaba en su primera ley, que la tasa de crecimiento del producto nacional estaba en función del crecimiento del sector manufacturero, ya que al generar rendimientos crecientes e incrementos de la productividad, se crean encadenamientos con los demás sectores de la economía.

En México el desmantelamiento de la política de industrialización y el inicio de la liberalización económica total empezó a mediados de los ochenta. La crisis de la deuda obligó al gobierno a asumir, ante las presiones de los organismos internacionales y los Estados Unidos, una serie de políticas de choque macroeconómico de contracción de la demanda agregada y de privatización de los activos productivos del Estado. A partir de la administración de Miguel de la Madrid (1982-1988), se implementaron políticas económicas liberales ${ }^{4}$, caracterizadas por la apertura comercial y la mínima participación del Estado en el mercado. Bajo esta premisa, el Estado empezó a desmantelar su política industrial, al abandonar el Programa Nacional de Fomento Industrial y Comercio Exterior (PRONAFICE), el cual garantizaba el tránsito gradual hacia un modelo de industrialización orientado hacia afuera. La estrategia en México, impulsada a partir de los años ochenta, se encuentra descrita en el Programa Nacional de Fomento Industrial y Comercio Exterior (PRONAFICE) del periodo 1984-1988, en el que se fijaron las líneas para impulsar a un sector industrial moderno. Las prioridades radicaban en la apertura comercial y promoción de las exportaciones. Durante el sexenio de Salinas de Gortari se abandonó totalmente todo esfuerzo de industrialización, y se dio el paso al proceso de desindustrialización temprana de la economía mexicana (Calderón y Hernández, 2016).

El abandono de las políticas de industrialización a favor de la apertura económica, a mediados de los ochenta, fue justificado por la teoría neoclásica del comercio internacional que argumentaba que con el libre comercio, cada país podría especializarse en la producción de bienes que produce más eficientemente y donde tiene abundancia de recursos (Krugman y Obstfeld, 2006). Bajo condiciones de libre comercio, se esperaba que el intercambio basado en la ventaja comparativa condujera a incrementar el consumo y producción mundial y por lo tanto el bienestar del consumidor. En un escenario de apertura y de competencia con los mercados internacionales, el crecimiento económico se concibió como resultado del impulso de la producción de exportaciones (Bhagwati y Krueger, 1973; Krueger, 1974; Balassa, 1978; Feder, 1983). El comercio internacional empezó a jugar un papel crucial en la definición de la política industrial.

4- El enfoque neoliberal asume que, en ausencia de trabas gubernamentales, los mercados son homogéneos e integrados; por lo tanto las señales del mercado fluyen fácilmente y de manera transparente. Expresado de otra forma, no se reconocen desequilibrios estructurales salvo los generados por intervenciones del Estado (Hernández y Lechuga, 1997). 


\section{Revisión de la literatura}

Trabajos recientes demuestran el fallo de la política comercial basada en el libre mercado aplicada en México, para incentivar el crecimiento económico. Sánchez y Moreno-Brid (2016), utilizan un modelo de cointegración para contrastar que el bajo dinamismo de las manufacturas ha implicado bajas tasa de crecimiento en México. Asimismo, Sánchez (2012) señala, mediante un modelo datos panel para los 32 estados de la República Mexicana, que el comportamiento de las manufacturas es fundamental para explicar las bajas tasas de crecimiento económico registradas de 1993 a 2010. En este contexto, algunos autores (Loría, 2009; Moreno-Brid y Ros, 2010; Sánchez, 2011; Calderón y Sánchez, 2012), atribuyen que el bajo crecimiento de los últimos años, se explica por la baja dinámica de las manufacturas, lo que ha llevado a cuestionarse el manejo de la política comercial actual. Algunos estudios resaltan los efectos negativos que ha traído consigo la postura del gobierno de reducir su participación en las actividades productivas del país, ya que al reducir la inversión en infraestructura ha ocasionado menor crecimiento de la economía (Moreno-Brid y Ros, 2010). Asimismo, para Tello (2007) es evidente que el cambio de modelo económico de fuerte participación a un modelo que favoreció el apoyo a empresas orientadas al mercado externo, fue la principal razón de que el crecimiento económico del país entrara a una fase de estancamiento. Para autores, el estancamiento económico de México está fuertemente ligado al reemplazo de una política industrial activa por una pasiva (Sánchez, 2010; Sánchez, 2013; Sánchez y Moreno Brid, 2016; y López, 2016).

La falta de crecimiento en México se explica por la incapacidad de las políticas de liberalización aplicadas, desde mediados de los ochentas, para impulsar el proceso de acumulación de capital. Nurkse (1955) explica que la raíz del problema radica en la falta de formación de capital, ya que es la que ocasiona que se genere un proceso circular que impide el crecimiento sostenido. El proceso circular que impide el crecimiento empieza con 1) un bajo nivel de ingreso, 2) escasa capacidad de ahorro y por ende de capital; lo que conduce a 3) baja productividad y por lo tanto a un bajo nivel de ingreso real per cápita. La formación de capital se entiende como un proceso en el que la sociedad destina parte su actividad productiva corriente a la generación de bienes de capital, los cuales incrementan la eficiencia del sector industrialmanufacturero. Esto significa que la industrialización debe ser concebida como un proceso de causalidad acumulativa, resultado de un aparato productivo fortalecido. En el caso de México, las políticas de liberalización comercial tan sólo favorecieron al proceso de acumulación de sus socios comerciales como los Estados Unidos y Canadá, en detrimento de su propio desarrollo económico nacional.

A partir del sexenio de Salinas de Gortari, y las subsecuentes administraciones presidenciales, el diseño de la política mantuvo la misma premisa. El Estado debía de reducir su papel activo como agente promotor del desarrollo, para dejar lugar al libre mercado. La política estaba basada en la apertura comercial indiscriminada, la estabilidad macroeconómica de los precios, para mejorar las ventajas comparativas, y atraer capital extranjero mediante políticas de privatización. Los diversos programas sexenales siguieron esta filosofía: el Programa Nacional de Modernización Industrial y Comercio Exterior (PRONAMICE) 1990-1994, el Programa de Política Industrial y Comercio Exterior (PROPICE) 1996-2000 y el Programa de Desarrollo Empresarial (PDE) 2006-2001. En todos estos programas el calificativo de "industrial" devino tan sólo en un recurso retórico sin un contenido real. Lo anterior implicó forzosamente que la 
llamada política "industrial" desapareciera y adquiriera un carácter meramente ficticio, y en realidad se sustituyera el término "industrial” por el "comercial". El Estado fue desplazado por el mercado; sus funciones se limitaron a crear las condiciones macroeconómicas necesarias para estabilizar los precios y garantizar el buen funcionamiento del "libre mercado" y del bienestar del consumidor. Con lo cual se desmantelo la política industrial real activa (Calderón y Sánchez, 2012). Esta política comercial disfrazada de "industrial" ha dado como resultado una dinámica de estancamiento económico en México, (Sánchez y Calderón, 2011), pues entre 1994 y 2014, la tasa de crecimiento promedio anual del PIB per cápita fue de $1.53 \%$.

En este contexto, nos tenemos que preguntar ¿de qué forma los mecanismos utilizados por la política comercial, entre 1995 y 2015, lograron tener un impacto sobre la formación de capital y la producción manufacturera? Por lo tanto, el objetivo de esta investigación es evaluar la efectividad de los mecanismos utilizados por la política comercial, basada en la estabilidad macroeconómica de precios y la liberalización económica, para lograr el aumento de la producción manufacturera, de las exportaciones y de la formación bruta de capital. Es decir, evaluar en qué medida la estabilidad de los precios, el tipo de cambio, la tasa de interés, las exportaciones manufactureras y la inversión extranjera directa contribuyeron a lograr los objetivos de la política comercial. Se asume que las variables vinculadas con el entorno internacional presentan efectos positivos sobre la "industrialización", entendida como el crecimiento del PIB manufacturero y la formación bruta de capital.

El artículo comprende la introducción, la revisión de literatura en el apartado uno, la descripción de la política industrial en el periodo de apertura comercial, que inicia a mediados de los ochenta en el apartado dos, la descripción de modelo econométrico utilizado para verificar la hipótesis así como la descripción de la variables en el apartado tres, la presentación de resultados y análisis de los principales hallazgos en el apartado cuatro y finalmente las conclusiones generales en el apartado cinco.

\section{La política “industrial” en México durante el periodo de apertura comercial}

La crisis de la deuda en la década de los ochenta, la presión de los organismos internacionales y de los Estados Unidos, obligaron a México a abandonar el modelo de política industrial orientada hacia el exterior (PRONAFICE) que le hubiera garantizado un transito gradual hacia la globalización. El proceso de industrialización por sustitución de importaciones (ISI) tuvo dos etapas. La primera, consistió en la sustitución de los bienes de consumo no duraderos que comprende entre 1946 y 1958, donde la producción se concentraba en este tipo de bienes. La segunda, de 1958 a 1981, constituyó el puntal de la expansión industrial con base en la sustitución de bienes de capital. A finales de los años setenta, se presentaron complicaciones para sustituir las importaciones de bienes de capital de alta tecnología, (Moreno Brid, Santamaría y Rivas Valdivia, 2009). Además durante el sexenio de López portillo (1976-1982), se petrolizó la economía, los ingresos fiscales y las exportaciones se hicieron dependientes de las exportaciones del petróleo. La renta petrolera se utilizó para endeudar al país, la economía sufrió los efectos desastrosos derivados de este proceso que culminó con la crisis de la deuda y la caída de los precios del petróleo. Se empezó a requerir mayor maquinaria y equipos sofisticados que debían ser comprados en el exterior, lo 
que implicaba mayor requerimiento de divisas; el resultado fue un constante aumento del déficit comercial que obligó al país a endeudarse en los mercados internacionales. Sin embargo, durante todo este periodo (1946-1981) México presentó una fase de crecimiento sostenido con una tasa promedio anual de crecimiento de 7 por ciento 5 .

A partir de los ochenta inició el proceso de desmantelamiento de la política industrial; la nueva clase política tecnocrática persiguió por todos medios, las reformas estructurales, desmantelar al Estado como promotor del desarrollo económico y convertirlo en el fiel guardián de las libres fuerza del mercado. El modelo económico impuesto desplazó a la ISI y se nutrió de las políticas neoliberales basadas en el Consenso de Washington (CW). Las políticas sugeridas por el CW se enmarcaban en una lógica de tipo Hayekiano según la cual toda intervención del Estado es nociva para el bienestar del consumidor. Este modelo fundó su política comercial en la versión más simplificada de las ventajas comparativas, la cual favorecía la apertura comercial indiscriminada, la desregulación y la estabilidad macroeconómica de los precios. Los organismos internacionales como el Fondo Monetario Internacional (FMI), el Banco Mundial y el Banco Interamericano de Desarrollo fueron los principales promotores de estas políticas a través del condicionamiento de los créditos otorgados a México.

El presidente Miguel de la Madrid Hurtado (1982-1988) inicia el proceso de apertura económica de manera unilateral, con la solicitud de admisión al Acuerdo General sobre Aranceles Aduaneros y de Comercio, GATT (por sus siglas en ingles) ${ }^{6}$ en 1985 y que obtuvo al siguiente año. En 1989, en el Plan Nacional de Desarrollo (PND), quedaron establecidas las bases bajo las cuales el país tendría que prepararse para competir en un mundo globalizado:

"Las líneas básicas para crecer son: la estabilización continúa de la economía; la ampliación de la disponibilidad de recursos para la inversión productiva, y la modernización económica, dicha estabilización se refiere a la estabilidad de precios no como un destino sino como un objetivo que requiere de esfuerzos permanentes. En cuanto a la necesidad de recursos, buscamos, más que una cifra fija, la ampliación continua de su disponibilidad para asegurar el crecimiento económico sostenido. La modernización es un proceso que no admite interrupción. Impone adaptar las estructuras económicas a las cambiantes condiciones por las que atraviesa el país y a las tendencias de la economía mundial. En este contexto, la política industrial y de comercio estará orientada a promover la modernización del aparato productivo, medidas de promoción a las exportaciones, así como de la Inversión Extranjera Directa como acceso a mercados, tecnología y empleos “, (Diario Oficial de la Federación, 1989:16).

Entre 1990 y 1994, la política industrial se implementó a través del Programa Nacional de Modernización Industrial y Comercio Exterior (PRONAMICE). Los principales objetivos estuvieron basados en una estrategia de orientar la industrialización y el crecimiento económico hacia la nueva coyuntura de globalización (Diario Oficial de la Federación, 1990). A partir de la administración de Miguel de la

5- Estimación propia con datos del Banco Mundial

6- Actualmente se le denomina Organización Mundial del Comercio (OMC). 
Madrid, el propósito de los planes nacionales de desarrollo radica en mantener la estabilidad macroeconómica y el control de la inflación, por ello cualquier diseño de política industrial a partir de entonces, quedó subordinado a estos propósitos. Por esta razón, a la política "industrial” subordinada a objetivos de estabilidad macroeconómica de precios, que no distingue entre los sectores productivos, ni consideraba los distintos efectos que pudieran tener se le denominó "política horizontal" (Arellano, 2014). En el cuadro 1 se presenta un resumen de las principales acciones en materia de política que se identificaron en los planes nacionales de desarrollo aplicados de 1990 a 2018.

\section{Cuadro 1. Programas nacionales establecidos para el periodo 1990 y 2018.}

\begin{tabular}{|c|c|c|}
\hline Periodo & Programas & Objetivos \\
\hline $\begin{array}{c}1990 \mathrm{a} \\
1994\end{array}$ & $\begin{array}{l}\text { Programa Nacional de Modernización Industrial y } \\
\text { Crecimiento Económico (PRONAMICE) }\end{array}$ & $\begin{array}{l}\text { Fortalecer el sector exportador. } \\
\text { Orientar las actividades de la industria hacia la } \\
\text { tendencia de globalización. }\end{array}$ \\
\hline $\begin{array}{c}1994 a \\
2000\end{array}$ & $\begin{array}{l}\text { Programa de Política Industrial y Comercio Exte- } \\
\text { rior (PROPICE) }\end{array}$ & $\begin{array}{l}\text { Impulsar las exportaciones. } \\
\text { Crear agrupamientos industriales regionales y } \\
\text { sectoriales. } \\
\text { Fomento del mercado interno y sustitución de } \\
\text { importaciones. }\end{array}$ \\
\hline $\begin{array}{l}2001 a \\
2006\end{array}$ & Programa de Desarrollo Empresarial (PDE) & $\begin{array}{c}\text { Mejorar la competitividad de las empresas en } \\
\text { especial de las micro, pequeñas y medianas em- } \\
\text { presas. } \\
\text { Apoyo focalizado a sectores que contribu- } \\
\text { yeran al desarrollo y creación de empleos. } \\
\text { Promoción de exportaciones. } \\
\text { Promoción de la IED. }\end{array}$ \\
\hline $\begin{array}{c}2007 a \\
2012\end{array}$ & $\begin{array}{l}\text { Comisión Mixta para la Promoción de las Exporta- } \\
\text { ciones (COMPEX) }\end{array}$ & $\begin{array}{l}\text { Promoción de las exportaciones. } \\
\text { Reducción de costos de transacción. Impulso de } \\
\text { la competitividad e innovación de las empresas. } \\
\text { Promoción de la IED. }\end{array}$ \\
\hline $\begin{array}{c}2013 a \\
2018\end{array}$ & $\begin{array}{c}\text { Programa Especial para Democratizar la Produc- } \\
\text { tividad (PDP) }\end{array}$ & $\begin{array}{c}\text { Promover el uso y asignación eficiente de los } \\
\text { factores de producción. } \\
\text { Elevar la productividad de trabajadores, empresas } \\
\text { y productores. } \\
\text { Mejorar las condiciones de competencia. } \\
\text { Elevar la productividad en las regiones y sectores } \\
\text { de la economía. }\end{array}$ \\
\hline
\end{tabular}

Fuente: Elaboración propia. 
Los argumentos del libre comercio aseguraban que la expansión del mercado, la atracción de tecnología y el aumento de las exportaciones serían los mecanismos para fortalecer a la industria manufacturera. Por ejemplo, en el PND del periodo 1995-2000, prevaleció el establecimiento de un programa de desregulación y simplificación administrativa, donde se estableció la necesidad de buscar nuevos mercados, de suscribir nuevos acuerdos comerciales con otros países, de promover una distribución más equitativa del ingreso, y elevar la competitividad (Diario Oficial de la Federación, 1995). En 1993, se logra firmar el Tratado de Libre Comercio con América del Norte (TLCAN), que implicó el mayor logro en materia de política comercial para México y que serviría para instrumentar una serie de programas que facilitarían la producción de exportaciones. En noviembre de 1995, se lanzó el Acuerdo para la Desregulación de la Actividad Empresarial, que señalaba que la llamada política industrial estaría enfocada a la desregulación, ya que los niveles de protección y regulación de ese entonces, desalentaban la producción, sobre todo de las pequeñas y medianas empresas.

Con la idea de orientar a la industria hacia la competitividad, el 31 de mayo de 1996 se instrumentó el Programa de Política Industrial y Comercio Exterior (PROPICE). En este programa se establecieron estrategias y líneas de acción que conformarían una política industrial integral y dinámica, cuyos objetivos radican en disminuir los costos de transacción y de información, ampliar las opciones tecnológicas, estimular el aprovechamiento de la dotación de los factores del país e impulsar una cultura de internacionalización (Diario Oficial de la Federación, 1996). Para lograr estos objetivos, se establecieron políticas horizontales para promover la competitividad de la industria, por ejemplo a través de programas de apoyo como el Programa de Importación Temporal para producir Artículos de Exportación (PITEX) y el Programa de Empresas Altamente Exportadoras (ALTEX). Mediante estos programas, el gobierno facilitaba el acceso a los mercados de América del Norte, a las empresas maquiladoras mediante la libre importación temporal de insumos y maquinarias para sus procesos productivos (Moreno-Brid y Ros, 2010).

Durante la administración de Vicente Fox (2000-2006) se profundizó la idea de que México debía continuar la estrategia de liberalización comercial, por ello se mantuvo la desregulación y simplificación administrativa. La implementación de programas orientados a la exportación continuó permitiendo la importación de insumos libre de impuestos destinados a la producción de bienes de exportación. La IED se trasformó en uno de los factores más importantes para el desarrollo industrial, por ello se hicieron modificaciones al marco legal para dar certidumbre a la inversión (Diario Oficial de la Federación, 2001). Durante el gobierno de Vicente Fox, la política industrial es reducida a una política empresarial que se plasmó en el Programa de Desarrollo Empresarial 2001-20067. En el programa es estableció como objetivo apoyar la competitividad de las pequeñas y medianas empresas (Pymes) ya que el propósito era convertir a las Pymes en un eje detonador del mercado interno (Presidencia de la República, 2001). Para ello, se llevaron a cabo acciones que facilitan el acceso al financiamiento, a la formación empresarial, a la innovación tecnológica e integración económica regional y sectorial (Brown y Domínguez, 2010).

7- El Programa de Desarrollo Empresarial, sustituye política industrial, por empresarial, en una visión gerencial que se explica por el papel central de la empresa como uno de los factores de la producción, sin embargo, los propósitos no se alcanzaron de manera efectiva al quedar supeditados al objetivo central de la política macroeconómica global de control de la inflación y estabilidad macroeconómica (Arellano, 2014). 
Durante la administración de Felipe Calderón (2007-2012) se puso en marcha la Comisión Mixta para la Promoción de las Exportaciones (COMPEX) para incentivar la actividad de la industria orientada al mercado exterior. Unos de los instrumentos fue la reducción de costos de transacción y disposiciones normativas que permitiera importar insumos libres de impuestos. También se mantuvo el fomento permanente para atraer y conservar a la IED, además se crearon esquemas de financiamiento entre el gobierno y los organismos intermedios para reforzar el desempeño de las Pymes en los estados (Diario Oficial de la Federación, 2007). Además se creó la figura de las Asociaciones Publico Privadas (APP) como un medio para semiprivatizar los bienes públicos. En este sexenio, al igual que en el anterior, se abrió de manera indiscriminada la economía a la IED, en particular se otorgaron entre el sexenio de Fox y Calderón más de 700 concesiones en el sector minero a las multinacionales extranjeras; además se inició la reforma del sector energético.

Para las empresas de menor tamaño fue difícil enfrentar la competencia global al no generar utilidades que les permitieran autofinanciarse. El mercado crediticio para la micro, pequeñas y medianas empresas se concentró en la banca privada; lo que ocasionó que sus créditos tuvieran mayores tasas de interés (generalmente la Tasa de Interés Interbancaria de Equilibrio (TIIE, + 12) y el monto exigido como colateral permaneciera alto. En este sentido a pesar del esfuerzo de los últimos gobiernos por incorporar a las pequeñas y medianas empresas en el escenario de competitividad al final, la labor recayó en la banca privada, dado que el Estado se mantuvo en la dinámica de implementar políticas pasivas (Pomar, Rendon y Rivera, 2011).

Finalmente para la administración del presidente Enrique Peña Nieto (2012-2018), en el Programa Nacional de Desarrollo 2013-2018 se plantea que el desarrollo se sustenta sobre la estabilidad macroeconómica y la apertura comercial. A diferencia de otras administraciones, en este plan se reconoce que se debe fortalecer en la misma medida el mercado doméstico y el mercado externo y apoyar a industrias incipientes que cuenten con ventaja comparativa. Sin embargo, estos objetivos se contraponen a la naturaleza de la llamada política industrial, ya que en el PND se le concibe como una herramienta para suministrar bienes públicos que permitan la expansión de la productividad y la producción y no como una política de intervención del Estado para otorgar subsidios y concesiones (Sánchez Juárez y Moreno Brid, 2016). Los mecanismos para lograr los objetivos del PND se encuentran en el Programa Especial para Democratizar la Productividad (PDP), donde se establecieron acciones para elevar la productividad laboral y de las empresas, incrementar el financiamiento de empresas con alto valor agregado, reducir los costos de transacción y mejorar las condiciones de competencia (Diario Oficial de la Federación, 2013). Durante el sexenio de Peña Nieto (2012-2018) se llevó a cabo la Reforma constitucional y la privatización total del sector energético. Durante este sexenio se liquidó y vendió al capital extranjero la petroquímica secundaria. También se creó la normativa que restringe la actividad productiva de PEMEX y la CFE, llamadas empresas productivas del Estado, a favor del capital extranjero. La modalidad de este proceso de privatización del sector petrolero radica en la concesión de contratos a las multinacionales para la explotación del petróleo que se encuentre en aguas profundas y someras. Contratos que "a priori" dan certidumbre y ventajas jurídicas a los capitales foráneos. Esta reforma no prevé de manera explícita ningún mecanismo de promoción de la industria nacional ni de transferencia de tecnología hacia ella.

La industrialización en México por medio de los automatismos del mercado nunca se concretó, ya que a pesar de la privatización prevalece la deficiencia estructural e insuficiencia dinámica de la acumulación de 
capital en el sector industrial (manufacturas), mientras que el comercio y los servicios han aumentado su importancia en la economía (Sánchez y Calderón, 2011). Las políticas “industriales” aplicadas en los últimos veinte años acentuaron el proceso de terciarización precoz de nuestra economía, el desempleo y la migración (ambos derivados de la absorción espuria del sector servicios y maquilador) como lo señalan Calderón y Hernández (2016). En este contexto, el país se mantiene en un estancamiento económico que se refleja en la tasa de crecimiento promedio anual del PIB per cápita, que fue de 1.53 por ciento ${ }^{8}$ entre 1994 y 2014 . Este promedio se encuentra abajo del 3 por ciento que se tendría en una situación de crecimiento acelerado (Rodrik, 2004)strategic lessons from the diverse experience with economic growth in last fifty years. The paper revolves around two key arguments. One is that neoclassical economic analysis is a lot more flexible than its practitioners in the policy domain have generally given it credit. In particular, first-order economic principleslu2014protection of property rights, market-based competition, appropriate incentives, sound money, and so onlu2014do not map into unique policy packages. Reformers have substantial room for creatively packaging these principles into institutional designs that are sensitive to local opportunities and constraints. Successful countries are those that have used this room wisely. The second argument is that igniting economic growth and sustaining it are somewhat different enterprises. The former generally requires a limited range of (often unconventional. En el grafico 1 se puede observar que las tasas de crecimiento del PIB manufacturero han mantenido un patrón similar a la tasa del PIB total, aunque ligeramente superiores. Cabe mencionar que hay dos años atípicos en este periodo, 2002 y 2003, en los cuales la tasa del PIB manufacturero presentó valores negativos mientras el PIB total incrementó ligeramente. Los años más críticos para la producción de manufacturas y donde se tuvieron tasas negativas de crecimiento coinciden con las crisis 1995, 2001 y 2009.

\section{Gráfico 1. Tasas de crecimiento del PIB manufacturero, total y per cápita}

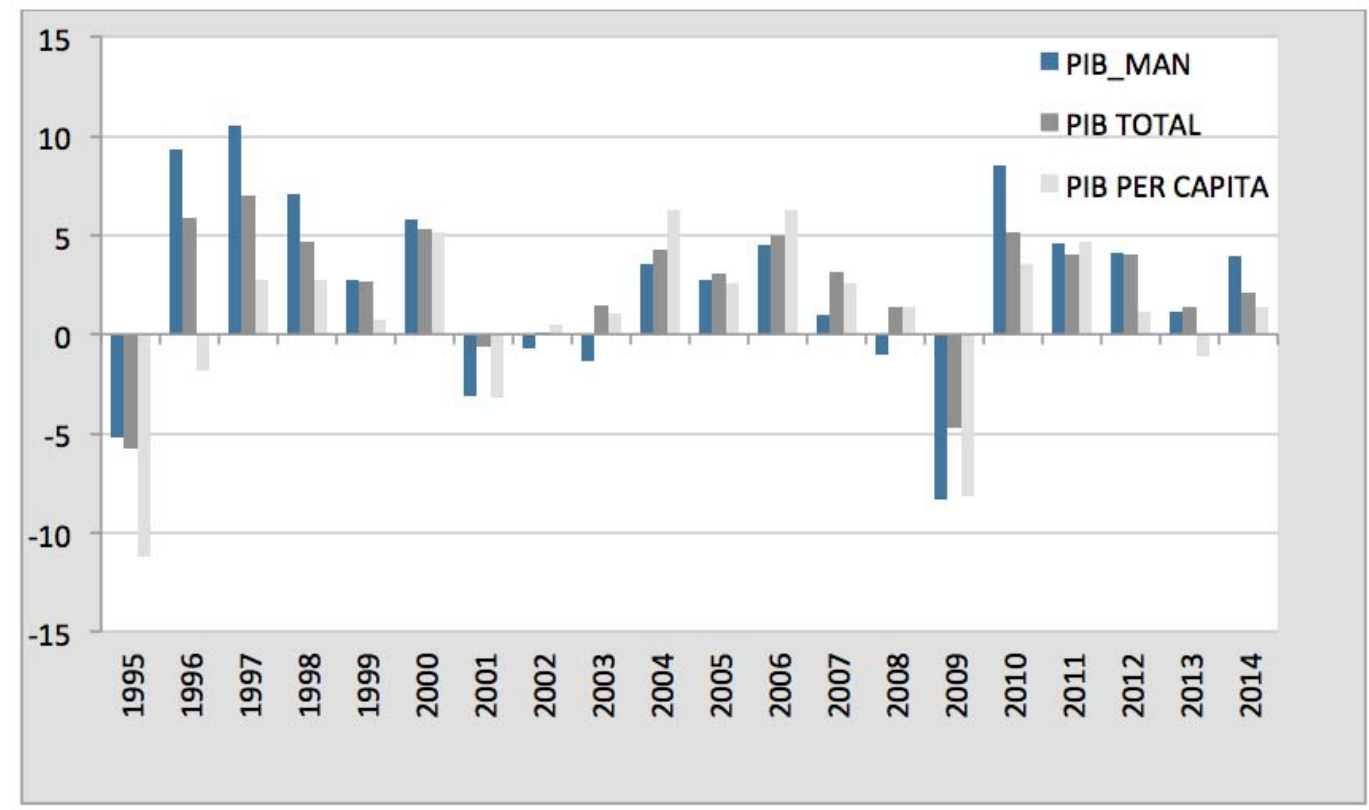

Fuente: Elaboración propia con datos de Banco de Información Económica de INEGI y Banco Mundial 
El resultado de este modelo de política "industrial" subordinado a la estabilidad macroeconómica de los precios, a la atracción de IED y a las privatizaciones, generó un aumento de mercancías vendidas en el exterior, pero con bajo valor agregado. Algunos estudios muestran que un modelo productivo basado en inversiones en maquila, cuya ventaja comparativa se basaba en los costos laborales, provocó que la productividad se estancara y por ende los salarios reales no aumentaran (Calva, 2007). Sin la existencia de un mercado interno con poder de compra y ahorro, la capacidad de generar inversión se dificultó. Shapiro (2007) encontró que, entre las razones del fracaso de la liberalización en países de Latinoamérica, se encuentra el hecho de que, a pesar del aumento de exportaciones, también lo hicieron las importaciones por la desintegración vertical del sector manufacturero. Si lo que se buscaba era aliviar los problemas de balanza de pagos, lo que se logró fue aumentar las importaciones a tasas mayores que las exportaciones, como lo sucedió en el caso de México (Moreno-Brid y Ros, 2010).

Rowthorn y Ramaswamy (1999) sostienen que una economía madura puede comenzar un proceso natural de desindustrialización como consecuencia de un dinámico y exitoso desempeño del sector manufacturero. En dicho escenario, se mantiene el crecimiento en la economía, el desempleo es bajo, el ingreso per cápita es elevado, la productividad del sector industrial es mayor que la del resto de los sectores, por lo tanto la demanda de servicios es creciente. Esto ocasiona un fenómeno natural de reasignación de recursos ligado la evolución de una economía madura, y que da lugar a una situación de desindustrialización. Sin embargo, para los países latinoamericanos este proceso de trasformación no cesó después de una cierta hegemonía del sector industrial, sino que dio paso a la tercerización precoz que trajo consigo la desindustrialización (Vera, 2009). Para el caso de México, Cruz (2015) identifica a 1981 como el año a partir del cual empieza un proceso de desindustrialización prematura, es decir, donde el sector manufacturero empieza a perder participación en el producto nacional y en el empleo total. Antes de 1981, el crecimiento de las manufacturas daba lugar a la acumulación del capital, al aumento de la productividad y de los ingresos, y todo ello eventualmente se reflejaba en efecto positivo para el resto de la economía de acuerdo a la primera ley de Kaldor. El debilitamiento de las manufacturas fue resultado de la implementación de políticas horizontales que buscaba apoyar un aparato productivo que lograra la competitividad en un entorno de libre mercado. En este proceso lo que se dio fue una mayor participación del sector terciario en la actividad de la economía. Entre 1988 y 1994, la tasa de crecimiento promedio anual (TCPA) del sector industrial fue de 3.9 por ciento, mientras que la de servicios fue de 4.1 por ciento (ver cuadro 2). Esta situación se reforzó para los siguientes años, por ejemplo entre 2008 y 2014, la TCPA del sector industrial fue de 1.6 por ciento y la de servicios fue de 3 por ciento.

\section{Cuadro 2. Tasas de crecimiento promedio anual del PIB industrial y de servicios (\%)}

\begin{tabular}{|ccc|}
\hline Periodo & Industrial & Servicios \\
$1988-1994$ & 3.9 & 4.1 \\
$1995-2001$ & 4.4 & 3.5 \\
$2002-2007$ & 2.2 & 3.3 \\
$2008-2014$ & 1.6 & 3.0 \\
\hline
\end{tabular}

Fuente: Elaboración propia con datos del Banco de Información Económica de INEGI 
Otra forma de observar el fenómeno de desindustrialización es a través de la baja proporción de la formación bruta de capital fijo como porcentaje del PIB. Durante el periodo de 1995 a 2014 dicha proporción se mantuvo en alrededor de 22 por ciento (Banco Mundial), cifra inferior al considerado por la CEPAL que es de 25 por ciento cuya proporción es la mínima necesaria para que un país pueda alcanzar tasas de crecimiento arriba de cinco por ciento (Moreno-Brid, 2013).

La disminución de la intervención del Estado en la economía en las últimas décadas fue promovida en todos los planes de desarrollo; sin embargo, algunos países asiáticos muestra que una política industrial con objetivos claros y de largo plazo puede producir resultados favorables en la senda de crecimiento de largo plazo. Por ejemplo, China mantiene altas tasas de formación bruta de capital fijo gracias al fuerte intervencionismo estatal. Algunas de las acciones emprendidas incluyen el otorgamiento de créditos a tasas subsidiadas, sujetas a objetivos de inversión definidos por el Gobierno, las exenciones impositivas y las políticas comerciales estratégicas (protección selectiva) para favorecer a los sectores orientados a la exportación. De esta manera la reciprocidad entre el apoyo estatal y la actuación del sector privado ha sido la clave para propiciar la acumulación de capital, que de otra forma no sería posible (Torija-Zane, 2012).

En resumen, en México la política industrial fue desmantelada a partir del sexenio de Salinas de Gortari. A lo largo de los años, diferentes administraciones federales fueron diseñando programas e instrumentos concretos para liberalizar la economía, abandonando el objetivo explícito de la industrialización como fundamento del desarrollo económico. Por esta razón, no es posible utilizar instrumentos concretos de política industrial puesto que dejaron de existir a partir del sexenio de Salinas. En este trabajo, consideramos al producto interno bruto de las manufacturas como proxy que mide el grado de industrialización inducido por "el mercado". Esto implica asumir teóricamente que la variación de la producción manufacturera determina al crecimiento de la economía. Como lo define la primera ley de Kaldor (Kaldor, 1966).

\section{Marco metodológico}

Para evaluar el impacto que tuvieron las políticas comerciales horizontales sobre el crecimiento del PIB manufacturero, la producción de exportaciones y la formación bruta de capital (usados como proxies del grado de industrialización), se emplearon métodos de análisis de series de tiempo. La conveniencia de utilizar un modelo de series de tiempo radica en que esta metodología nos evita determinar a priori la relación causa-efecto de las variables y permite que se presenten relaciones bi direccionales entre ellas, también es posible medir los efectos sesgados de las variables pues el modelo es de tipo dinámico (y no estático). En específico, se estimaron vectores de corrección de error (VECs) para considerar la endogeneidad de las variables. En su forma general el VEC se especifica como (Patterson, 2000):

$$
\Delta y_{t}=\Pi y_{t-1}+\Gamma_{1} \Delta y_{t-1}+\cdots+\Gamma_{p-1} \Delta y_{t-p+1}+u_{t}
$$

Donde: $\mathrm{y}=$ vector de variables endógenas, $\Pi=-\left(I_{k}-A_{1}-\cdots-A_{p}\right)$ y $\Gamma_{i}=-\left(A_{i+1}+\cdots+A_{p}\right)$ para $i=1, \ldots$ p-1. $\Delta$ =denota primeras diferencias y $\mu_{t}=$ vector de residuos con características de ruido blanco.

El VEC proviene de la estimación de un vector autoregresivo (VAR) en niveles al sustraer $y_{t-1}$ de ambos lados de la igualdad. Debido a que todas las variables tienen un orden de integración máximo de 
I(1), $\Delta y_{t}$ no contiene una tendencia estocástica por lo que el término $\Pi y_{t-1}$ es el único que contiene variables $I(1)$. Si esto es cierto, $\Pi y_{t-1}$ debe ser $I(0)$ y debe contener las relaciones de cointegración. De esta forma, tenemos que el VEC proporciona los términos $\Gamma_{i}(j=1, \ldots, p-1)$ que contienen los parámetros de corto plazo, y representa el parámetro de largo plazo (Lütkepohl y Krätzig, 2004).

Dado que estamos considerando la inclusión de variables exógenas al modelo, la especificación de (1) debe incluir un vector de variables estocásticas $Z_{t}$ con $B$ como su respectiva matriz de parámetros: $\Delta y_{t}=\Pi y_{t-1}+\Gamma_{1} \Delta y_{t-1}+\cdots+\Gamma_{p-1} \Delta y_{t-p+1}+B z_{t}+u_{t}$

Para nuestro caso, el vector de variables endógenas y exógenas consideradas fueron las siguientes:

$$
y_{t}=\left[\begin{array}{c}
P I B_{-} M A N \\
F B K \\
X_{-} M A N
\end{array}\right] \quad z_{t}=\left[\begin{array}{c}
I N P C \\
I T C R \\
T I R \\
I E D
\end{array}\right]
$$

Donde la variable PIB_MAN corresponde al PIB manufacturero, FBK es la formación bruta de capital fijo, X_MAN son exportaciones manufactureras, INPC es un índice de precios al consumidor, ITCR es un índice del tipo de cambio real, TIR es una tasa de interés real, IED es la inversión extranjera directa. Por lo tanto tenemos que, para capturar el efecto de las políticas comerciales horizontales utilizamos como proxy a la variable PIB manufacturero, pues representa el principal objeto gobierno. El modelo también permite captar a través de la formación bruta de capital fijo y de las exportaciones manufactureras el efecto de las estas políticas, que están especificadas como variables endógenas. Los instrumentos de política que consideramos como explicativos son variables que representan los resultados de la aplicación de varios instrumentos a lo largo de los años. Para medir el impacto de la estabilidad macroeconómica, se consideró la fluctuación de los principales precios de la economía: del índice nacional de precios al consumidor (inflación), del tipo de cambio peso dólar y de la tasa de interés de los CETES. Aunque se reconoce que estas variables fueron controladas por la política monetaria y por lo tanto podrían considerarse endógenas, el propósito de asumir su exogenidad radica en que nuestro objetivo es medir el efecto que la política monetaria restrictiva tuvo sobre la producción, la inversión y las exportaciones. Se espera que la baja fluctuación de estas variables incremente la inversión y la producción de mercancías, ya que mantiene los costos bajos. Para analizar el efecto de la política de apertura comercial, se consideró la producción de exportaciones pero como una variable endógena en el sistema, ya que existe un proceso de retroalimentación con las manufacturas y la formación bruta de capital. También se consideró el efecto de la política de estímulo a la inversión extranjera a través de la IED, la cual se asume es exógena, pues responde principalmente a planes estratégicos de empresas transnacionales (Allard Neumann, 2011).

Todas las variables que se encontraban en unidades monetarias fueron deflactadas con año base 2008, fueron desestacionalizadas ${ }^{9}$ y se convirtieron a logaritmos naturales. Los datos estadísticos provienen del Banco de Información Económica del Instituto Nacional de Estadística y Geografía (Instituto Nacional de Estadística y Geografía, 2016) y del Banco México (Banxico, 2016). El periodo utilizado fue de 1995 a $2015^{10}$ con periodicidad trimestral.

9- Para su desestacionalización se empleó el método de Census X-12

10- La selección del periodo estuvo en función de dos aspectos: 1) es que con el fin de contrastar los efectos de los instrumentos de promoción hacia la industria cuya dirección recae en el libre mercado y apertura comercial, en dónde el mayor grado de integración se presentó tras la firma del TLCAN, y 2) por la disposición de datos y frecuencia. 
Para conocer la naturaleza estacionaria de las series, primero se realizaron las pruebas de raíz unitaria Dickey Fuller Aumentada (DFA) y la de Phillips-Perron (P-P), que se presentan en el cuadro 3. Como se puede observar, a excepción de la IED, todas las variables son estacionarias en primeras diferencias, lo que implica que es necesario establecer si las tres variables endógenas comparten o no una relación de largo plazo, es decir, si están cointegradas.

Cuadro 3. Estadísticos de las pruebas de raíz unitaria DFA y P-P. (Probabilidades en paréntesis).

\begin{tabular}{|c|c|c|c|c|c|c|}
\hline \multirow[t]{3}{*}{ Variable } & \multicolumn{2}{|c|}{ Niveles } & \multicolumn{2}{|c|}{ Niveles } & \multicolumn{2}{|c|}{ Primeras diferencias } \\
\hline & \multicolumn{2}{|c|}{ Tendencia y constante } & \multicolumn{2}{|c|}{ Constante } & \multicolumn{2}{|c|}{ Constante } \\
\hline & DFA & $\mathbf{P}-\mathbf{P}$ & DFA & P-P & DFA & $\mathbf{P}-\mathbf{P}$ \\
\hline \multirow[t]{2}{*}{ PIB_MAN } & $-3.74 * *$ & -2.26 & -1.16 & -1.65 & $-8.33^{* * *}$ & $-8.38^{* * *}$ \\
\hline & $(0.024)$ & $(0.440)$ & $(0.446)$ & $(0.45)$ & $(0.000)$ & $(0.000)$ \\
\hline \multirow[t]{2}{*}{ FBK } & -1.94 & -1.99 & -2.51 & -2.42 & $-8.63 * * *$ & $-8.75^{* * *}$ \\
\hline & $(0.630)$ & $(0.596)$ & $(0.120)$ & $(0.139)$ & $(0.000)$ & $(0.000)$ \\
\hline \multirow[t]{2}{*}{ X_MAN } & -2.08 & -1.94 & 0.31 & 1.152 & $-10.46 * * *$ & $-10.48^{* * *}$ \\
\hline & $(0.550)$ & $(0.630)$ & $(0.980)$ & $(0.997)$ & $(0.000)$ & $(0.000)$ \\
\hline \multirow[t]{2}{*}{ INPC } & -3.2 & $-14.8^{* * *}$ & -1.69 & $-8.61^{* * *}$ & $-3.51^{* * *}$ & $-8.17^{* * *}$ \\
\hline & $(0.090)$ & $(0.000)$ & $(0.430)$ & $(0.000)$ & $(0.010)$ & $(0.000)$ \\
\hline \multirow[t]{2}{*}{ ITCR } & -2.97 & -2.93 & $-3.41 * *$ & $-3.29 * *$ & $-7.73^{* * *}$ & $-7.80 * * *$ \\
\hline & $(0.150)$ & $(0.160)$ & $(0.013)$ & $(0.019)$ & $(0.000)$ & $(0.000)$ \\
\hline \multirow[t]{2}{*}{ TIR } & -3.45 & -2.77 & -2.47 & -1.81 & $-8.39 * * *$ & $-8.43 * * *$ \\
\hline & $(0.052)$ & $(0.21)$ & $(0.13)$ & $(0.37)$ & $(0.000)$ & $(0.000)$ \\
\hline \multirow[t]{2}{*}{ IED } & $-8.768^{* * * *}$ & $-8.77^{* * *}$ & $-8.37^{* * *}$ & $-8.40 * * *$ & \multirow{2}{*}{ n.n } & \multirow{2}{*}{ n.n } \\
\hline & $(0.000)$ & $(0.000)$ & $(0.000)$ & $(0.000)$ & & \\
\hline
\end{tabular}

Fuente: Elaboración propia

Nota: Los asteriscos ${ }^{* *} \mathrm{y}^{\star *}$ indican el rechazo de la hipótesis nula a nivel de significancia de $1 \%$ y $5 \%$ respectivamente. n.n.= No necesario

Para determinar si existe cointegración entre PIB_MAN, FBK y X_MAN se empleó la prueba de Johansen (Johansen, 1991) y Johansen y Joselius (Johansen y Juselius, 1990), la cual implica estimar un modelo VAR no restringido con las series endógenas en niveles. Para la selección de los rezagos en los modelos de series de tiempo se siguió el siguiente procedimiento: primero con base en los criterios de selección de rezagos se eligió el menor rezago sugerido, segundo, se aplicaron pruebas a los residuales de estabilidad matemática, de correlación serial (LM), de heterocedasticidad (White) y de normalidad (Jarque-Bera). Si los residuales no satisfacían alguna de estas pruebas, se aumentaba el número de rezagos hasta que los residuales pasaran todas las pruebas. Por lo tanto, con este procedimiento se eligieron 8 rezagos para realizar la prueba de cointegración. Los resultados se presentan en el cuadro 4, donde el estadístico de traza indica la existencia de un vector de cointegración, esto implica que esta relación de largo plazo debe considerarse en la estimación del modelo. 


\section{Cuadro 4. Prueba de cointegración de Johansen Variables: PIB_MAN, FBK y X_MAN}

\begin{tabular}{|c|c|c|c|c|}
\hline \multicolumn{5}{|l|}{ Prueba de traza } \\
\hline $\mathrm{H}_{0}$ : ecuaciones de cointegración & Eigenvalor & Estadístico de trazo & Valor crítico 0.05 & Prob. \\
\hline Ninguna & 0.2153 & $31.709 * *$ & 29.797 & 0.029 \\
\hline Máximo 1 & 0.1617 & 13.519 & 15.494 & 0.097 \\
\hline Máximo 2 & 0.0037 & 0.284 & 3.841 & 0.594 \\
\hline \multicolumn{5}{|l|}{ Prueba de máximo eigenvalor } \\
\hline $\mathrm{H}_{0}$ : ecuaciones de cointegración & Eigenvalor & $\begin{array}{l}\text { Estadístico Eigen- } \\
\text { Max }\end{array}$ & Valor crítico 0.05 & Prob. \\
\hline Ninguna & 0.2153 & 18.189 & 21.131 & 0.123 \\
\hline Máximo 1 & 0.1617 & 13.235 & 14.264 & 0.072 \\
\hline Máximo 2 & 0.0037 & 0.284 & 3.841 & 0.594 \\
\hline
\end{tabular}

Fuente: Elaboración propia.

Se consideran 8 rezagos en la estimación.

** Denota el rechazo de la hipótesis nula a un nivel de significancia de $5 \%$.

\section{Análisis de los resultados}

Establecida la existencia de cointegración, la estimación del modelo VEC (2) se realizó con una variable exógena (estimación A) y con todas las variables exógenas (estimación B). La razón es mostrar que con el aumento de variables la estimación del modelo mejora y los coeficientes son robustos. Cada estimación requirió utilizar un numero diferente de rezagos para asegurar que el modelo pasara las pruebas de diagnóstico a los residuales. De esta forma, tenemos que la mejor estimación corresponde al modelo que incluye a todas las variables exógenas (estimación B). En el cuadro 5 se presentan los resultados solamente de los coeficientes de la ecuación de largo plazo y de las exógenas, ya que los coeficientes de las variables endógenas rezagas se evalúan con la prueba de Granger. 
- Revista de Ciencias Sociales y Humanidades. ISSN-P: 0188-9834 ISSN-E:2395-8669.

Cuadro 5. Estimación del VEC con cinco rezagos. Periodo: 1995:01-2015:04.

\begin{tabular}{|c|c|c|c|c|c|c|}
\hline $\begin{array}{c}\text { Ecuación de Cointegra- } \\
\text { ción }\end{array}$ & Estimación & A & & Estimación & B & \\
\hline PIBMAN(-1) & 1 & & & 1 & & \\
\hline \multirow[t]{2}{*}{ FBK(-1) } & 0.318 & & & 0.218 & & \\
\hline & $(0.191)$ & & & $(0.167)$ & & \\
\hline \multirow[t]{2}{*}{ X_MAN(-1) } & -0.809 & & & $-0.612 * *$ & & \\
\hline & $(0.268)$ & & & $(0.230)$ & & \\
\hline Constante & -16.352 & & & -15.569 & & \\
\hline Corrección de error & DPIB_MAN & $\triangle \mathbf{F B K}$ & DX_MAN & DPIB_MAN & $\triangle \mathbf{F B K}$ & DX_MAN \\
\hline \multirow{2}{*}{ CointEq1 } & $-0.097 * *$ & $-0.096 * *$ & $0.111 * *$ & $-0.113^{* *}$ & $-0.143^{* *}$ & $0.083 * *$ \\
\hline & $(0.027)$ & $(0.035)$ & $(0.052)$ & $(0.032)$ & $(0.039)$ & $(0.045)$ \\
\hline \multirow[t]{2}{*}{ C } & $0.013^{* *}$ & $-0.039 * *$ & 0.001 & -0.052 & $-0.189 * *$ & 0.119 \\
\hline & $(0.004)$ & $(0.005)$ & $(0.007)$ & $(0.069)$ & $(0.087)$ & $(0.098)$ \\
\hline \multirow{2}{*}{$\triangle \mathrm{INPC}$} & $-0.351^{* *}$ & $-0.502 * *$ & 0.092 & -0.337 & $-0.530 * *$ & 0.391 \\
\hline & $(0.217)$ & $(0.284)$ & $(0.418)$ & $(0.223)$ & $(0.278)$ & $(0.313)$ \\
\hline \multirow{2}{*}{$\triangle \mathrm{ITCR}$} & & & & -0.047 & 0.021 & $0.550 * *$ \\
\hline & & & & $(0.061)$ & $(0.075)$ & $(0.085)$ \\
\hline \multirow{2}{*}{ DTIR } & & & & 0.027 & 0.026 & 0.024 \\
\hline & & & & $(0.019)$ & $(0.024)$ & $(0.027)$ \\
\hline \multirow{2}{*}{ IED } & & & & 0.006 & $0.019 * *$ & -0.01 \\
\hline & & & & $(0.062)$ & $(0.008)$ & $(0.008)$ \\
\hline \multicolumn{7}{|l|}{ Pruebas a la estimación } \\
\hline $\mathbf{R}^{2}$ & 0.421 & 0.434 & 0.351 & 0.434 & 0.498 & 0.663 \\
\hline $\mathrm{R}^{2}$ Ajustada & 0.257 & 0.274 & 0.167 & 0.235 & 0.322 & 0.545 \\
\hline F-statistic & 2.564 & 2.710 & 1.907 & 2.183 & 2.827 & 5.613 \\
\hline \multicolumn{7}{|l|}{ Pruebas a los residuos } \\
\hline Autocorrelación (LM) & & rezagos & Prob. & & rezagos & Prob. \\
\hline Prueba LM & 12.136 & 5 & 0.206 & 8.534 & 5 & 0.481 \\
\hline Heterocedasticidad & & df & Prob. & & df & Prob. \\
\hline Prueba White & 193.193 & 204 & 0.696 & 228.648 & 240 & 0.690 \\
\hline Normalidad & & df & Prob. & & df & Prob. \\
\hline Jarque-Bera & 8.318 & 6 & 0.216 & 4.290 & 6 & 0.638 \\
\hline Estabilidad matemática & \multicolumn{3}{|c|}{ Se satisface condición. } & \multicolumn{3}{|c|}{ Se satisface condición. } \\
\hline
\end{tabular}

Fuente: Elaboración propia.

Errores estándar se encuentran en paréntesis.

** Denota la significancia estadística a 5\% de probabilidad. 
Los resultados indican que ningún mecanismo establecido por los programas nacionales de industrialización tuvo efecto estadísticamente significativo sobre el crecimiento del producto manufacturero. Por ejemplo, la estabilización de precios, medida a través del INPC no contribuyó a estimular el crecimiento de la producción. La promoción de inversión extranjera tampoco tuvo efecto sobre el PIB manufacturero. El hecho de que la IED no muestre un efecto significativo, puede explicarse por la implementación de políticas de atracción de inversión extranjera sin restricciones y sin efectos de arrastre en los procesos de producción nacional, ya que las ventajas comparativas se basaron en la abundancia de mano de obra poco calificada. El resultado fue la proliferación de establecimientos cuyo propósito era el ensamble de mercancías, con reducida capacidad para aumentar la productividad del trabajo y por lo tanto de los salarios. Es así, que la IED no muestra incidencia en la tasa de crecimiento de las manufacturas. Esto revela que las condiciones de estabilidad de precios y apertura económica que hasta ahora han mantenido los diversos PND no son suficientes y quizás lo que han hecho es contribuir a la desindustrialización de México (Cruz, 2015).

Las estimaciones muestran que, algunos mecanismos de política comercial, si tuvieron efectos sobre el crecimiento de la inversión fija bruta. Por ejemplo, la variable de los precios (INPC) fue estadísticamente significativa, lo que implica que a medida que disminuye la inflación, la tasa de crecimiento de la FBK aumenta aproximadamente en 0.52 por ciento. También la IED fue significativa, por lo que su tasa de crecimiento ocasiona que la tasa de la FBK aumente en 0.18 puntos porcentuales. Aunque la estabilidad de los precios fue un factor importante para el capital, la tasa de interés no tuvo efecto. Si bien, la teoría argumenta que una disminución en la tasa de interés induce un efecto positivo para los empresarios mediante el deseo de una mayor inversión a través de la adquisición de capital, para la industria mexicana esto no se pudo comprobar. Consideramos que la explicación reside en que, después de la transición hacia un régimen comercial liberalizado y desregulador, la desaparición de la banca de desarrollo como promotor de inversión, y el surgimiento de los bancos privados transnacionales como principal fuente de financiamiento, ocasionó una contracción de los préstamos para actividades productivas. En este contexto, la falta de créditos hacia el sector productivo se ha convertido en un obstáculo para la inversión (Moreno-Brid y Ros, 2010).

Con el fin de contrastar los efectos de corto plazo en las variables endógenas se aplicó la prueba de causalidad de Granger. Esta prueba permite conocer si cambios en una variable están precedidos por los cambios en otra variable. En el cuadro 6 se presentan los resultados.

Cuadro 6. Prueba de causalidad Granger

\begin{tabular}{|c|c|c|}
\hline A) & \multicolumn{2}{|c|}{ Variable dependiente: DPIB_MAN } \\
\hline Excluye: & Chi-cuadrada & v \\
\hline$\Delta$ FBK & 7.342 & 0.196 \\
\hline DX_MAN & 6.044 & 0.302 \\
\hline B) & \multicolumn{2}{|c|}{ Variable dependiente: $\Delta$ FBK } \\
\hline Excluye: & Chi-cuadrada & Prob. \\
\hline DPIB_MAN & 17.081 & $0.004^{* * *}$ \\
\hline DX_MAN & 12.105 & $0.033^{* *}$ \\
\hline C) & Variable dependiente: DX_MAN \\
\hline Excluye: & Chi-cuadrada & Prob. \\
\hline DPIB_MAN & 18.142 & $0.003^{* * *}$ \\
\hline DFBK & 4.402 & 0.493 \\
\hline
\end{tabular}

Fuente: Elaboración propia

Los asteriscos ${ }^{* *} \mathrm{y}^{* *}$ denotan la significancia estadística a 1 y $5 \%$ de probabilidad. 
En el bloque A se muestra que ni las variaciones en la formación bruta de capital ni en las exportaciones preceden a las variaciones en la producción manufacturera. En el bloque B, los resultados indican que la producción manufacturera y la tasa de crecimiento de las X_MAN causan en el sentido Granger, cambios en tasa de la FBK, dado que se rechaza la hipótesis nula de no causalidad. En el bloque C, se muestra que solo la tasa de crecimiento de la producción manufacturera causa en el sentido Granger a la tasa de crecimiento de las exportaciones. En este sentido, los programas que se instrumentaron para estimular la producción de exportaciones lograron que los productos manufacturados sean parte integral de las exportaciones.

La crisis económica mundial desencadenada en 2008 sirvió para llamar la atención sobre la vulnerabilidad de los naciones frente al entorno global. Por ello, en la agenda para el desarrollo 2030 elaborada por la ONU en 2015, se establecieron entre los objetivos lograr el crecimiento económico sostenible y el trabajo decente, así como promover la industrialización inclusiva y sostenible. Sin embargo, como lo han hecho notar varios autores, hasta la fecha México mantiene una posición liberal de no intervención estatal en la economía y ello se ha hecho evidente con la introducción de reformas estructurales en el ámbito laboral, hacendario, energético y financiero que dificultan significativamente alcanzar los objetivos de la agenda 2030 (Trejo Nieto, 2017).

\section{Conclusión}

Fundada en políticas horizontales cuyos ejes principales fueron la apertura comercial, la estabilización macroeconómica y el funcionamiento del libre mercado; la política industrial liberal, de naturaleza pasiva, trajo consigo la aplicación de programas gubernamentales que buscaban el progreso espontaneo de la industria y la competitividad con base en la atracción de los capitales extranjeros. Bajo este contexto, en este artículo se buscó probar si algunos de los mecanismos utilizados por los programas de política industrial liberal de corte horizontal, lograron los objetivos establecidos de favorecer la industrialización de México entre 1995 y 2015.

La evidencia empírica indica que la política de estabilización de precios, que ha tomado prioridad en la agenda de las últimas administraciones en cuestión de política industrial, no ha contribuido a mejorar el desempeño del crecimiento de la producción manufacturera. Sin embargo, para la formación bruta de capital, la política de estabilización de precios tuvo un impacto positivo, lo que se puede atribuir a que en una economía global el control sobre los precios y costos (salarios) permite que los inversionistas extranjeros se enfrenten a condiciones favorables para adquirir bienes de producción. Si bien la estabilidad de precios fue significativa para la acumulación del capital, no lo fue para la producción manufacturera en general.

Las estimaciones del modelo empírico muestran que la estabilidad del tipo de cambio, y la tendencia hacia su apreciación, contribuyó para mantener los objetivos de inflación, pero no fomentó directamente la industrialización. Lo que sí logró fue tener un impacto positivo sobre el crecimiento de las exportaciones manufactureras, ya que permitió reducir los costos de importación de insumos necesarios para la producción de este sector. Con respecto a la hipótesis de que los instrumentos de la apertura, basados en la promoción de las exportaciones, tuvieron efectos significativos sobre la tasa de crecimiento del PIB 
manufacturero y la formación bruta de capital, los resultados de integración indican que comparten una trayectoria de crecimiento de largo plazo. Sin embargo, no existen efectos de corto plazo del crecimiento de las exportaciones sobre el crecimiento del PIB manufacturero, pero donde si existen indicios de causalidad de las exportaciones es sobre el crecimiento de la formación de capital.

La apertura económica trajo consigo el incremento de inversión extranjera directa, sin embargo, los resultados muestran que esa inversión no ha generado un impacto significativo sobre el crecimiento del PIB manufacturero o las exportaciones. Si bien, la IED se ha concentrado en el sector manufacturero, la composición de la producción con poco contenido nacional, ha llevado a que el impacto sobre el valor total de la producción no sea considerable. Donde sí ha mostrado efectos significativos fue sobre el crecimiento de la FBK, por lo que consideramos que permitió aumentar el capital en el país independientemente de las derramas que posteriormente hubiera creado.

En general, la implementación de programas federales que estarían orientados a la modernización de un sector industrial, no ha sido exitosa. Los efectos parciales que presentan cada una de variables de industrialización muestran que la política liberal industrial, de carácter pasivo, subordinada a las políticas horizontales, no fue efectiva para promover la inversión y producción requeridas para aumentar las tasas de crecimiento económico. Esto nos lleva a concluir que es necesaria la implementación de una política industrial activa, con el fin de fortalecer el mercado interno con un sector manufacturero que sea capaz de impulsar un crecimiento económico sostenido. Consideramos que, en las condiciones actuales, la estrategia del libre mercado parece ser menos convincente para asegurar el crecimiento económico de México. El éxito de algunos países asiáticos donde el sector privado ha contado con el apoyo del Estado para identificar oportunidades del comercio internacional, podrían ser ejemplos para retomar en el futuro.

\section{Referencias}

Allard Neumann, Raúl. 2011. Las empresas multinacionales en la globalización : relaciones con los Estados. Estudios Internacionales, 40 (158): 59-99. DOI: https://doi.org/10.5354/0719-3769.2007.14154.

Amsden, Alice H. 1994. Why isn't the whole world experimenting with the East Asian model to develop?: Review of the East Asian miracle. World Development, 22 (4): 627-633. DOI: https://doi. org/10.1016/0305-750X(94)90117-1.

Arellano, Mario. 2014. Apertura Externa, Industria Manufacturera y Politica Industrial En Mexico: Vision Prospectiva. México: Palibrio.

Balassa, Bela. 1978. Exports and economic growth. Journal of Development Economics, 5 (2): 181-189. DOI: https://doi.org/10.1016/0304-3878(78)90006-8.

1982. Development Strategies in Semi-Industrial Economics. Baltimore: Johns Hopkins Uni-

versity Press.

Banxico. 2016. Banco de México. Estadísticas. http://www.banxico.org.mx/estadisticas/index.html (28 de agosto, 2016).

Bhagwati, Jagdish N. y Anne O. Krueger. 1973. Exchange Control, Liberalization, and Economic Development. American Economic Review, 63 (2): 419-427. 
Brown, Flor y Lilia Domínguez. 2010. Políticas e instituciones de apoyo y mediana empresa en México. En Políticas de apoyo a las pymes en América Latina. Entre avances innovadores y desafíos institucionales, editado por C Ferraro y G Stumpo. Naciones Unidas Comisión Económica para América Latina y el Caribe (CEPAL), 299-342. http://repositorio.cepal.org/handle/11362/2560 (15 de marzo, 2017).

Calderón, Cuauhtemoc y Leticia Hernández. 2016. Cambio Estructural y Desindustrialización en México. Panorama Económico, 11 (23): 153-190.

Calderón, Cuauhtemoc e Isaac Sánchez. 2012. Crecimiento económico y política industrial en México. Revista Problemas del Desarrollo, 43 (170): 125-154. Universidad Nacional Autónoma de México.

Calva, José Luis. 2007. Política industrial manufacturera. México: Porrúa y UNAM.

Cruz, Moritz. 2015. Premature de-industrialisation: theory, evidence and policy recommendations in the Mexican case. Cambridge Journal of Economics, 39 (1): 113-137. DOI: https://doi.org/10.1093/cje/ beu036.

Diario Oficial de la Federación. 1989. Plan Nacional de Desarrollo 1989-1994. http://dof.gob.mx/index. php? year $=1989 \&$ month $=05 \&$ day $=31$.

1990. Programa Nacional de Modernización Industrial y Comercio Exterior. México. http://www.dof.gob.mx/nota_detalle.php?codigo=4642383\&fecha=24/01/1990 (29 de marzo, 2017). 1995. Plan Nacional de Desarrollo 1995-2000. México. http://www.diputados.gob.mx/ LeyesBiblio/compila/pnd.htm.

1996. Programa de Política Industrial y Comercio Exterior. México. http://dof.gob.mx/ nota_detalle.php? codigo $=4887082 \&$ fecha $=31 / 05 / 1996 \&$ print $=$ true.

2001. Plan Nacional de Desarrollo 2001-2006. México. http://www.diputados.gob.mx/ LeyesBiblio/compila/pnd.htm.

2007. Plan Nacional de Desarrollo 2007-2012. México. http://www.diputados.gob.mx/ LeyesBiblio/compila/pnd.htm.

2013. Programa para Democratizar la Productividad 2013-2018. México: Diario Oficial de la Federación.

http://www.dof.gob.mx/nota_detalle.php?codigo=5312422\&fecha=30/08/2013.

Feder, Gershon. 1983. On exports and economic growth. Journal of Development Economics, 12 (1-2): 59-73. DOI: https://doi.org/10.1016/0304-3878(83)90031-7.

Fishlow, Albert. 1994. Miracle or design? : lessons from the East Asian experience. Washington D.C.: Overseas Development Council.

Hernández, Guillermo. y Jesús. Lechuga. 1997. Teoría económica de las sociedades periféricas : reflexiones sobre el desarrollo económico. México: Universidad Autónoma Metropolitana, Unidad Iztapalapa, División de Ciencias Sociales y Humanidades.

Instituto Nacional de Estadística y Geografía. 2016. Banco de Información Económica. Banco de Información Económica. http://www.inegi.org.mx/sistemas/bie/.

Johansen, Soren. 1991. Estimation and Hypothesis Testing of Cointegration Vectors in Gaussian Vector Autoregressive Models. Econometrica, 59 (6): 1551-1580. DOI: https://doi.org/10.2307/2938278.

Johansen, Søren y Katarina Juselius. 1990. Maximum Likelihood Estimation and Inference On Cointegration - With Applications to the Demand for Money. Oxford Bulletin of Economics and Statistics, 52 (2): 169-210. DOI: https://doi.org/10.1111/j.1468-0084.1990.mp52002003.x. 
Kaldor, Nicholas. 1966. Causes of the slow rate of economic growth of the United Kingdom: an inaugural lecture. London: Cambridge University Press.

Krueger, Anne. 1974. The Political Economy of the Rent-Seeking Society. American Economic Review, 64 (3): 291-303.

Krugman, Paul R. y Maurice. Obstfeld. 2006. Economía internacional : teoría y política. Madrid: Person Education.

Lall, Sanjaya, Manuel Albaladejo y Maurício Moreira. 2004. Latin American industrial competitiveness and the challenge of globalization. Washington, DC: Institute for the Integration of Latin America and the Caribbean IDB-INTAL.

Little, I. M. D., Tibor. Scitovsky y Maurice FitzGerald. Scott. 1970. Industry and trade in some developing countries: a comparative study. London: Published for the Development Centre of the Organization for Economic Co-operation and Development by Oxford U.P.

López-Gallardo, Julio. 1999. Es posible acelerar el crecimiento de América Latina. Revista de Economía Contemporánea, (5): 133-156.

López, Laura Ivonne. 2016. La política industrial y apertura comercial en México. Tesis de maestría en economía, Tijuana: El Colegio de la Frontera Norte.

Loría, Eduardo. 2009. Sobre el lento crecimiento económico de México. Una explicación estructural. Investigación Económica, 68 (270): 37-69.

Lütkepohl, Helmut. y Markus Krätzig. 2004. Applied time series econometrics. Cambridge University Press. Moreno-Brid, Juan Carlos. 2013. Industrial Policy: A Missing Link in Mexico's Quest for Export-led Growth. Latin American Policy, 4 (2): 216-237. DOI: https://doi.org/10.1111/lamp.12015.

Moreno-Brid, Juan Carlos y Jaime Ros. 2010. Desarrollo y crecimiento en la economía mexicana : una perspectiva histórica. México: Fondo de Cultura Económica.

Moreno Brid, Juan Carlos, Jesús Santamaría y Juan Carlos Rivas Valdivia. 2009. Manufactura y TLCAN: un camino de luces y sombras. Economía, UNAM, 3 (8): 95-114.

Nurkse, Ragnar. 1955. Problemas de formación de capital en los países insuficientemente desarrollados. México: Fondo de Cultura Económica.

Patterson, Kerry. 2000. An introduction to applied econometrics : a time series approach. Hampshire: MacMillian Press.

Pomar, Silvia, Araceli Rendon y Martin Rivera. 2011. Política Industrial de Apoyo para el Desarrollo de la Micro, Pequeña y Mediana Empresa. En Ante la sucesión presidencial: Propuestas de política económica y social para el nuevo gobierno, editado por UAM. México, 25.

Presidencia de la República. 2001. Presentación del Programa de Desarrollo Empresarial 2001-2006. Discurso. http://fox.presidencia.gob.mx/actividades/?contenido=2245 (29 de marzo, 2017).

Rodrik, Dani. 2004. Growth Strategies. Harvard University. http://www.ksg.harvard.edu/rodrik/ (29 de marzo, 2017).

Rowthorn, Robert y Ramana Ramaswamy. 1999. Growth, trade, and deindustrialization. IMF Staff Papers, 46 (1). https://www.imf.org/external/pubs/ft/staffp/1999/03-99/rowthorn.htm (28 de marzo, 2017). Sánchez, Isaac. 2010. Estancamiento económico e industrias manufactureras regionales en México, 19932010: explicación y propuestas. Tesis de doctorado en ciencias sociales con especialidad en estudios regionales, Tijuana: El Colegio de la Frontera Norte. 
- Revista de Ciencias Sociales y Humanidades. ISSN-P: 0188-9834 ISSN-E:2395-8669.

Sánchez, Isaac. 2011. Estancamiento económico en México, manufacturas y rendimientos crecientes: un enfoque kaldoriano. Investigación Económica, 70(277): 87-126.

Sánchez, Isaac. 2012. Ralentización del crecimiento y manufacturas en México. Nóesis. Revista de Ciencias Sociales y Humanidades, 21(41): 137-172. DOI: https://doi.org/10.20983/noesis.2012.1.6

Sánchez, Isaac. 2013. Política industrial activa como estrategia para el crecimiento de la economía mexicana. Cuadernos de Trabajo de la UACJ. Estudios Regionales en Economía, Población y Desarrollo, 15: 1-37. Sánchez, Isaac y Cuauhtemoc Calderón. 2011. Apertura, inestabilidad y estancamiento económico en México. En Integración de México en el TLCAN: sus efectos sobre el crecimiento, la restructuración productiva y el desarrollo económico, editado por Cuauhtemoc Calderón y Víctor Cuevas. México: Porrúa, 19-49.

Sánchez, Isaac y Juan Moreno-Brid. 2016. El reto del crecimiento económico en México: industrias manufactureras y política industrial. Revista Finanzas y Política Económica, 8 (2): 271-299. DOI: https:// doi.org/10.14718/revfinanzpolitecon.2016.8.2.4.

Shapiro, Helen. 2007. Industrial policy and growth. New York. www.un.org/esa/desa/papers/2007/ wp53_2007.pdf (29 de marzo, 2017).

Tello, Carlos. 2007. Estado y desarrollo económico : México 1920-2006. México: Universidad Nacional Autónoma de México.

Torija-Zane, Edgardo. 2012. Desarrollo industrial y política macroeconómica de los dragones asiáticos: 1950-2010. Santiago de Chile.

Trejo Nieto, Alejandra. 2017. Crecimiento económico e industrialización en la agenda 2030: Perspectivas para México. Problemas del Desarrollo, 48 (188): 83-111. DOI: https://doi.org/10.1016/j.rpd.2017.01.005. Vera, Leonardo. 2009. Cambio estructural, desindustrialización y pérdidas de productividad: evidencia para Venezuela. Cuadernos del Cendes, 26 (71): 89-115. 